\title{
EXEMPLIFICATION OF CATALYST DESIGN FOR MICROWAVE SELECTIVE HEATING and its APPLICATION TO EFFICIENT IN SITU CATALYST SYNTHESIS
}

\author{
Kaiyang Wang, Georgios Dimitrakis* and Derek J. Irvine* \\ Department of Chemical and Environmental Engineering, Faculty of Engineering, \\ University of Nottingham, Nottingham, NG7 2RD, UK; \\ *Corresponding authors email: georgios.dimitrakis@nottingham.ac.uk and \\ derek.irvine@nottingham.ac.uk
}

Keywords: microwave, selective heating, organometallic catalysts

\begin{abstract}
The use of dielectric spectroscopy to develop an underpinning understanding of the molecular transformations involved in achieving the successful, rapid in situ synthesis of a catalytic chain transfer polymerisation (CCTP) catalyst using microwave heating is reported. The hypothesis behind the molecular design of this catalyst, such that it was tailored towards the application of microwave heating $(\mathrm{MWH})$, is discussed, reviewed relative to the empirically results and compared to the performance of a benchmark preformed catalyst. The overall number/type of function group present in the catalyst, the degree of flexibility exhibited by its organic ligand system and level of solvation achieved are shown to be key factors affecting the interaction between the catalyst and the applied microwave energy. Use of microwave heating leads to fast, in situ formation of the catalyst (less than 30 second) within the polymerisation mixture, rendering prepreparation steps unnecessary and ensuring it is generated prior to the polymerisation reaction commencing. The data also suggests catalysts' synthesis is achieved at levels of microwave power as low as 5 Watts, further adding to the efficiency and sustainability of the method and presents a potentially enormous opportunity to intensify current industrial processes.
\end{abstract}




\section{Introduction}

The last few decades have witnessed a significant increase in the number of methods and known mechanisms that can be used to control polymerisation reactions of all types. In the particular case of free radical chemistry, the control methods have been reported include: Nitroxide Mediated Polymerisation (NMP) [1], Atom Transfer Radical Polymerisation (ATRP) [2], Reversible Addition Fragmentation Polymerization (RAFT) [3], and Catalytic Chain Transfer Polymerisation (CCTP) [4]. The advent of these new control methodologies has allowed the polymer using community to gain access to many new polymer molecular structural types that were previously unobtainable.

Amongst these new materials, the ability to obtain oligomers, i.e. polymers with relative low molecular weight (i.e. $<10,000 \mathrm{~g} \mathrm{~mol}^{-1}$ ), has generated significant interest. The aim of developing these species is to provide novel materials that deliver specific improved application performance in fields such as: coatings, surfactants, dispersants, emulsifiers, etc. [5-7]. In the specific cases of free radically produced oligomers, the control method that is often most appropriate to use to synthesise such moieties is CCTP $[4,8]$. In a typical CCTP reaction, low spin cobalt (II) organometallic catalysts are utilised to control the molecular weight and the polydispersity (Đ) of the polymer by acting as chain transfer agents (CTA). A CTA prematurely terminates the growth of one polymer chain (thus controlling its molecular weight), but keeps the radical "alive" such that it can initiate the growth of new chain (hence ensuring a high yield). It is the catalytic nature of these CCTP control agents that enables the facile synthesis of very low molecular weight oligomers when using very low levels of CTA, typically parts per million (ppm). 
Currently, the most frequently cited of these cobalt CTA's is bis-[(difluoroboryl) diphenylglyoximato] cobalt (II) (PhCoBF), because it exhibits the best balance between activity and stability. However, it is synthesised in a two-step process that is not highly atom efficient. The first step involves the reaction of two mole equivalents of the diphenylglyoxime (DPG) ligand with cobalt (II) acetylacetonate to form cobaloxime which contains 2 bidentate DPG ligands (See Figure 1, structure (a)).

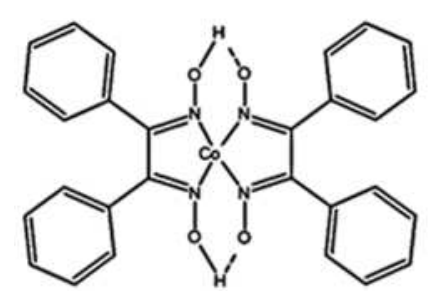

(a)

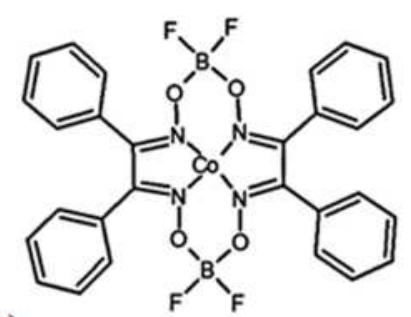

(b)

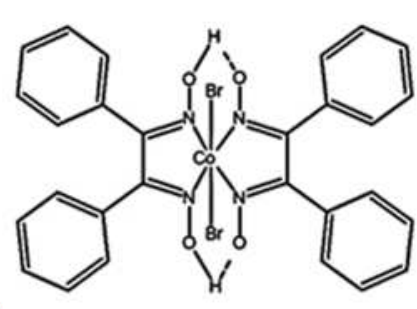

(c)

Figure 1. Molecule structure of: (a) Cobaloxime, (b) PhCoBF and (c) $\mathrm{CoBr}_{2} \mathrm{DPG}_{2}$

This procedure has also been reported with the dimethylglyoxime version of the ligand (DMG) and both of these products have been shown to be active CCTP catalysts in their own right. However, they are sensitive to being deactivated by oxidation and/or hydrolysis. The second step involves reacting this step 1 product with boron trifluoride diethyl etherate (BF.OEt 2 ) to generate a single tetradentate ligand as shown in Figure 1Figure 1, (structure (b)) $[9,10]$. This enhances the complexes' stability towards oxidation and hydrolysis and allows it to be handled readily in air in solid form. However, the yield from this second stage is typically only in the region of $40 \%$ and so requires purification stages to be added to the manufacturing process, increasing both the cost and the environmental footprint of the process [8].

Consequently, the authors have investigated the synthesis of alternative catalysts that are more economically and atom efficient. In previous journal publications, the authors have reported a successful adaptation of the existing PhCoBF synthesis, which 
generates the cobalt halide/glyoxime catalyst of the type shown in Figure 1(structure (c)) [11]. The bromide member of this family has been shown to demonstrate levels of activity and stability similar to those of $\mathrm{PhCoBF}$, but it is synthesised using a single step and without the need to be ring closed to achieve the greater level of stability. Thus, this catalysts is significantly more atom efficient, sustainable and potential industrial applicable than PhCoBF. Furthermore, adopting a single stage process potentially enables the use of in situ catalyst manufacture and continuous flow processing. To do so would further increase the sustainability and intensification of the process, because it reduces waste by; (a) removing the catalyst pre-preparation process form the overall manufacturing cycle, (b) eliminating catalyst deactivation during storage and (c) minimising inter-batch cleaning of the polymerisation etc.

However, to successfully achieve a free radical polymerisation process that utilises in situ catalyst synthesis, it must be ensured that the catalyst synthesis has been achieved within the polymerisation medium prior to the onset of initiation to produce the radicals. This is of particular importance when low concentrations of reagents are being used, such as in the case of catalytic CTA usage, because it must be ensured that diffusion issues do not retard generation of the control agent. The application of microwave (or dielectric) heating (MWH) methods is known to accelerate reaction processes by achieving either/both rapid/uniform and selective heating [12]. This is because with MWH methods, energy is transferred by directly coupling the dipoles within the target material with an applied alternating electromagnetic (EM) field. Therefore, the dielectric properties ( $\varepsilon^{\prime}$ : dielectric constant and $\varepsilon "$ : dielectric loss) of a material determine the ability of a specific material to both; interact with/store energy from such an alternating field in the case of $\varepsilon^{\prime}$, and then convert an amount of the stored energy into heat with regard to $\varepsilon$ '. When a mixture of several different materials is placed 
within an EM field, the material with the higher dielectric properties typically has a greater potential to couple with the alternating field, and thus undergo selective heating [13]. The existence of selective heating has already been reported on metal catalysts such as Fe [14, 15], Sn [16], and Co [10].

Prior work by the authors has empirically demonstrated that applying MWH to the in situ CCTP process can accelerate the rate of the combined in situ catalyst generation and polymerisation process by utilising selective MWH $[11,16]$. This paper investigates the interactions between the metal complexes and ligands discussed above with an EM field. The aims are to assess how the selective heating changes as the ligands coordinated to the cobalt centre are modified, develop a fundamental understanding of the mechanisms that MWH influences these transformations and enable the design of improved catalysts structures which maximise the benefits of MWH. This work would also prove that the catalysts have been synthesised prior to the polymerisation commencing and demonstrate that dielectric spectroscopy can be a facile tool to aid in defining mechanistic behaviour in complex mixtures.

\section{Materials and methods}

\subsection{Materials}

Cobalt (II) bromide (98\%, Aldrich), PhCoBF (Dupont), dimethylglyoxime (98\%, Aldrich), diphenylglyoxime (98\%, Aldrich), and cyclohexanone (98\%, Aldrich) were all used as supplied without further purification.

\subsection{Dielectric property measurements}

The dielectric properties of all samples were measured using the cavity perturbation technique. The equipment and methods used are detailed in prior publications $[17,18]$. In this study, the cavity resonated in the $\mathrm{TM}_{050}$ transverse magnetic mode at a frequency 
of $2470 \mathrm{MHz}$. The cavity perturbation technique is ideally suited in measuring accurately small quantities of materials because it centres on measuring small perturbations in the resonant characteristics of standing modes inside a hollow metal cavity of predetermined geometry and does require the elaborate calibration procedures to be conducted prior to measurement. Dielectric property measurements were conducted on cyclohexanone solutions of all organometallic complexes used in this study. Since the molecular concentrations of the organometallics in the solvent can potentially have an effect in the overall dielectric response of the solvent/organometallic solution, care was taken to ensure that the molar concentration of each organometallic in the solvent was maintained constant.

\subsection{Microwave heating trials}

All heating experiments were conducted in a single mode Sairem Miniflow microwave reactor (maximum power $200 \mathrm{~W}$ ). In order to ensure maximum transfer of microwave (MW) power into the sample, the impedance of the sample was matched to that of the MW source by the use of a stub tuner and a moving short circuit plate. An optical fibre temperature sensor was introduced directly into the solution bulk via a septum to accurately gauge the bulk temperature of the bulk of the sample. A cylindrical choke was fitted to the reactor (at the point of sample insertion) to arrest any microwave leakage. All heating experiments were carried out at a frequency of $2450 \mathrm{MHz}$ which is a standard frequency allocated for laboratory experiments in the Industrial Scientific and Medical (ISM) band.

\subsection{In situ Synthesis of organic liganded catalysts $\mathrm{Co}_{2} \mathrm{Br}_{2} \mathrm{DPG}_{2}$ and $\mathrm{Co}_{2} \mathrm{Br}_{2} \mathrm{DMG}_{2}$}

In a typical synthesis following the methods described in previous publications $[10$, 19], cobalt (II) bromide $(0.036 \mathrm{~g}(0.11 \mathrm{mmol})$ and $0.053 \mathrm{~g}(0.22 \mathrm{mmol})$ DPG or $0.025 \mathrm{~g}$ (0.22mmol) DMG (i.e. a mole ratio of 1:2) were added to the selected solvent (40 mL, 
$386 \mathrm{mmol})$ with stirring at room temperature. A dark green $\left(\mathrm{Co}_{2} \mathrm{Br}_{2} \mathrm{DPG}_{2}\right)$ and light green $\left(\mathrm{Co}_{2} \mathrm{Br}_{2} \mathrm{DMG}_{2}\right)$ solution was obtained as the in situ catalyst being formed.

\section{Results and discussion}

\subsection{Dielectric property measurements of precursors and catalyst mixed with solvent}

An assessment of dielectric properties of cyclohexanone and mixtures of this solvent containing the precursors and final catalysts was conducted to aid in understanding/predicting the interactions between EM field and the materials within these multi component solutions. Comparison of the loss tangent ( $\tan \delta$ ) values, which is defined as the ratio of the dielectric loss factor $(\varepsilon " ')$ and the dielectric constant $(\varepsilon ')$, was used to predict the relative capability of the materials contribute to the heating of the medium and/or enhancement of a chemical transformation when introduced to an EM field. It was proposed that adding new dipoles and an increased level of solubilisation of the metal complex by adding organic ligands, should lead to an increase in the dielectric properties of the reaction media that contain the organometallic components. Moreover, when comparing the organically liganded catalysts, it was also postulated that introducing both increased flexibility and additional functional groups into the complex by "replacing" the tertadentate ligand in $\mathrm{PhCoBF}$ with the bidentate equivalents in $\mathrm{CoBr}_{2} \mathrm{DPG}_{2}$ would lead to the latter exhibiting greater dielectric properties. Consequently, this would improve the latter complexes' ability to translate the energy absorbed from the EM field into molecular motion and hence lead to the promotion of subsequent heating/chemical transformations [20, 21]. Figure 2 details the trend in the tan $\delta$ values for the cyclohexanone solvent and mixtures of solvent and catalyst/precursor over the temperature range from $20^{\circ} \mathrm{C}$ to $140^{\circ} \mathrm{C}$. 


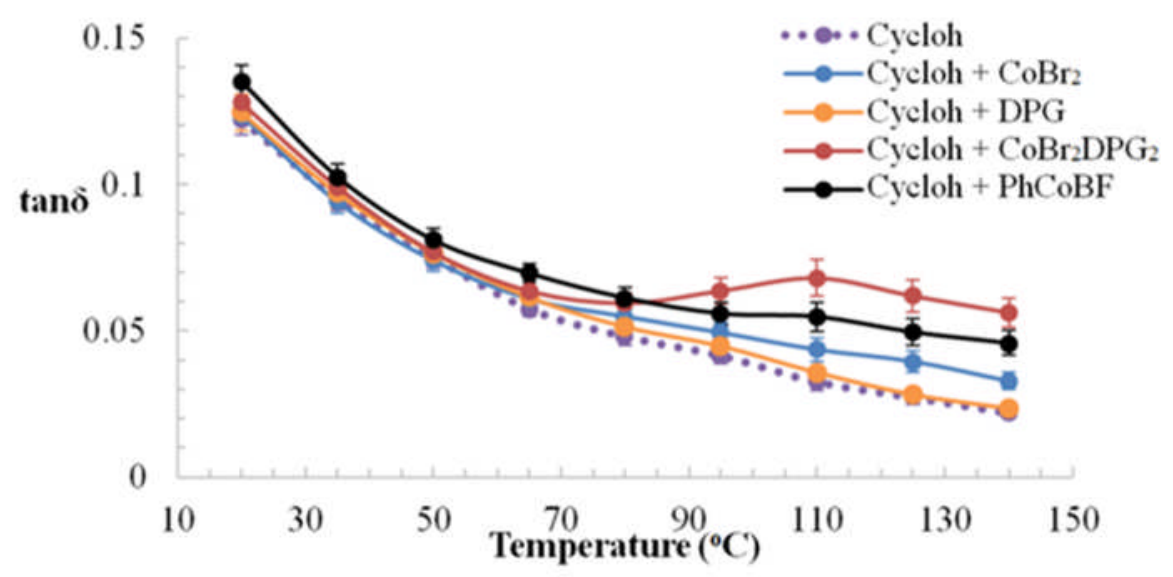

Figure 2. Plot of the temperature dependence of $\tan \delta$ of cyclohexanone (cycloh) and cyclohexanone solutions of different catalysts/precursors at a concentration of 240 ppm.

From Figure 2, it was observed that the tan $\delta$ value for all samples follows similar trend below $65^{\circ} \mathrm{C}$. Closer inspection of the data showed that, mixing the ligand into the solvent alone did not change the dielectric properties of the solution at the $240 \mathrm{ppm}$ level. However, in all cases, the addition of the organometallic complexes to cyclohexanone produced measureable increases in $\tan \delta$ even at this very low concentration. Furthermore, above $65^{\circ} \mathrm{C}$ the values of $\tan \delta$ of the solution containing the organometallic additives were noted to exhibit increased deviation from both: (a) the behaviour of the solvent and solvent ligand mixture and (b) from one another. This demonstrated that all the organometallic precursors should undergo selective $\mathrm{MWH}$ at these low concentrations and potentially contribute to the heating of these mixtures and/or the promotion of a chemical transformation within that medium. Furthermore, the influence that specific cobalt complexes would potentially be more differentiated above $65^{\circ} \mathrm{C}$, where the magnitude of the $\tan \delta$ value between the various metal containing additives were noted to be greater. This is also of potential importance in catalyst choice/design because the target polymerisation has a typical reaction temperature of $80^{\circ} \mathrm{C}$. Inclusion of the organic liganded catalysts raised the tan $\delta$ values to 
a greater extent $\left(113 \%\right.$ for $\mathrm{CoBr}_{2} \mathrm{DPG}_{2}, 92 \%$ for $\mathrm{PhCoBF}$, where these figures are the increases relative to the value exhibited by the solvent alone) than did the cobalt halide precursors $\left(34 \%\right.$ for $\left.\mathrm{CoBr}_{2}\right)$. This data supported the initial hypothesis that adding these organically liganded metal catalysts into a solvent should enhance the solutions' overall ability to interact with EM energy and so undergo stronger MWH. This was attributed to the combination of increased solvation of the metal centre and the introduction of additional dipoles into the structure upon the addition of the organic soluble ligands. The data also supported the proposal that different ligand structures affected the dielectric properties to different levels, because the $\tan \delta$ of bidentate $\mathrm{CoBr}_{2} \mathrm{DPG}_{2}$ was indeed shown to be greater than that of the more rigid tetradentate molecular structure of $\mathrm{PhCoBF}$. This effect was linked to the fact that the coordinated $\mathrm{CoBr}_{2} \mathrm{DPG}_{2}$ /cyclohexanone structure is more flexible and so the dipoles in the structure are freer to align with the E-field orientation of the EM energy. This also suggested that the $\mathrm{CoBr}_{2} \mathrm{DPG}_{2}$ may prove to be a more efficient $\mathrm{CTA}$ at $80^{\circ} \mathrm{C}$ in a $\mathrm{MWH}$ polymerisation due to its greater dielectric properties.

Inspection of the results in $\varepsilon^{\prime}$ in Figure 3, suggested from the trend in $\varepsilon$ ' suggested that all samples/mixtures exhibited very similar interaction with the EM field in terms of the energy that can be stored in their structure across the full temperature range. The trend in $\varepsilon^{\prime}$ with temperature reveals that the total polarisation of all the samples/mixtures is dominated by the presence of cyclohexanone with the catalyst and precursors having only a minor effect, which was attributed to the very small amounts of catalyst precursors present into the mixture. 

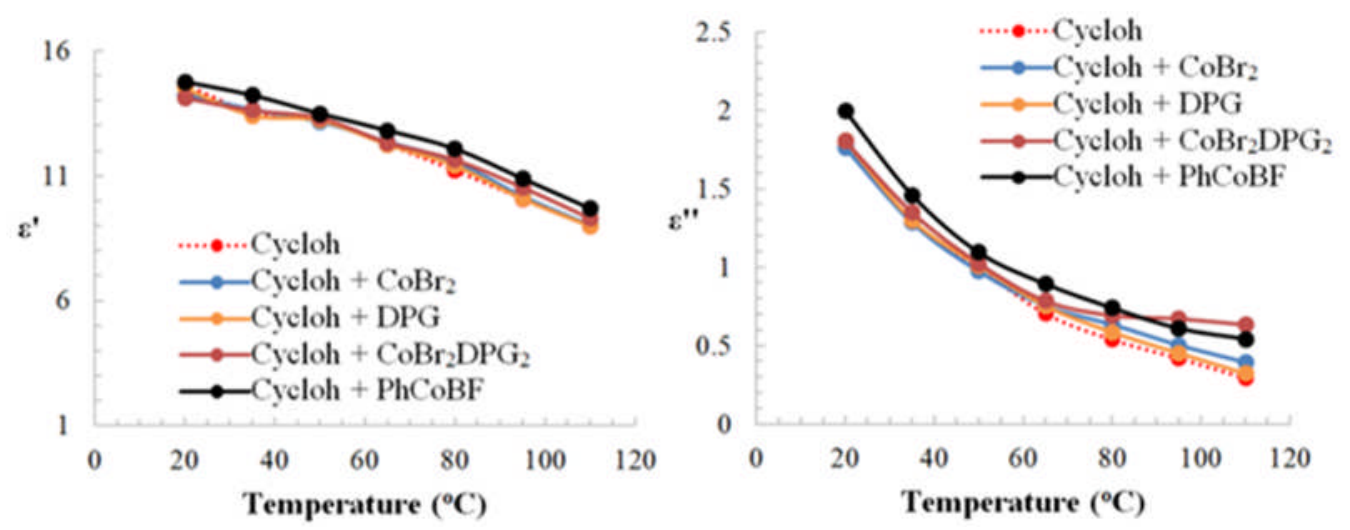

Figure 3. Plot of the temperature dependence of dielectric constant ( $\left.\varepsilon^{\prime}\right)$ and loss factor ( $\left(\varepsilon^{\prime \prime}\right)$ of cyclohexanone (cycloh) and mixture of different catalysts/precursors.

By contrast, the $\varepsilon$ " value exhibited similar trends to that of $\tan \delta$, i.e. increased values when the organometallic species were present, especially so when above $65^{\circ} \mathrm{C}$ where significant differences were noted. The behaviour of $\varepsilon$ "' indicates that the presence of the organometallic species (even in small amounts) contributes to the total amount of the stored energy that can be converted into heat in the samples/mixtures. The increased differentiation above $65{ }^{\circ} \mathrm{C}$ was attributed to the additional compatibility with the medium (solvation) and the greater flexibility enabling more facile molecular motion. Thus, again would predict that the designed $\mathrm{CoBr}_{2} \mathrm{DPG}_{2}$ would be the better choice of catalyst at the typical polymerisation temperature of $80^{\circ} \mathrm{C}$.

Furthermore, review of the trends in the dielectric data suggested that the most effective temperature range for these solutions to be influenced by the EM energy is below $40^{\circ} \mathrm{C}$. This is because the values of all the dielectric properties are higher in this region, so predicting their maximum interaction with EM energy. Thus, this study of the dielectric data would predict that in a MWH reaction: (a) the catalyst should be synthesised readily via MWH below $40^{\circ} \mathrm{C}$ ensuring that it is in place prior to the 
polymerisation commencing and that our designed $\mathrm{CoBr}_{2} \mathrm{DPG}_{2}$ should be the preferred catalyst in a free radical polymerisation at $80^{\circ} \mathrm{C}$.

Table 1 shows a comparison of the dielectric properties measured at $20^{\circ} \mathrm{C}, 80^{\circ} \mathrm{C}$ and $100^{\circ} \mathrm{C}$ of the premade $\mathrm{PhCoBF}$, in situ $\mathrm{CoBr}_{2} \mathrm{DPG}_{2}$, MMA monomer and the low molecular weight oligomers that would be the potential products from a target continuous polymerisation process to generate functional oligomeric products. $80^{\circ} \mathrm{C}$ is typical of an industrial batch polymerisation with $\mathrm{AIBN}$ as the initiator, whilst $100^{\circ} \mathrm{C}$ is the upper temperature because is the boiling point for the MMA monomer. Furthermore, assessment of the dielectric properties allowed the penetration depth (PD) to be calculated. The PD defines the depth that which the microwave power falls to $1 / \mathrm{e}$ of its power at sample surface (i.e. reduce to 0.368 of its original value). This gives an indication of the heat distribution within the sample, to ensure even heating through the reaction mixture and the vessel size should not exceed 2 PD lengths In this study the reaction vessel was $3 \mathrm{~cm}$ in diameter so there were no issues with the depth is in these experiments penetration depth (see Table S1 in ESI document for the calculated penetration depths at these temperatures).

From the review of the data in Table 1 is clear that the loss tangent of the organically liganded catalysts is higher than that of the monomer. Therefore, they would be predicted to be selectively heated relative to the rest materials as the reaction commences because this point, the reaction system will be largely monomer.

Table 1. $\tan \delta$ for the organically liganded catalysts and MMA and cyclohexanone at $80^{\circ} \mathrm{C}$ and $110^{\circ} \mathrm{C}$.

\begin{tabular}{ccccc}
\hline \multirow{2}{*}{ Entry } & Material & $\tan \delta$ & $\tan \delta$ & $\tan \delta$ \\
& & $20^{\circ} \mathrm{C}$ & $80^{\circ} \mathrm{C}$ & $110^{\circ} \mathrm{C}$ \\
\hline 1 & methyl methacrylate & 0.079 & 0.030 & N/A \\
2 & cyclohexanone & 0.122 & 0.048 & 0.032 \\
3 & MMA Dimer & 0.34 & 0.144 & 0.100 \\
4 & MMA Trimer & 0.089 & 0.26 & 0.250 \\
\hline
\end{tabular}




\begin{tabular}{lcccc}
\hline 5 & MMA Tetramer & 0.016 & 0.108 & 0.175 \\
6 & MMA Pentamer & 0.007 & 0.036 & 0.070 \\
7 & Initiated Dimer radical & N/A & 0.190 & 0.210 \\
8 & $\mathrm{CoBr}_{2} \mathrm{DPG} \mathrm{F}_{2}$ & 0.128 & 0.065 & 0.068 \\
9 & $\mathrm{PhCoBF}^{2}$ & 0.135 & 0.065 & 0.055 \\
\hline
\end{tabular}

However, the $\tan \delta$ of the oligomers suggest that this may not be the case when a significant amount of product oligomer has been produced, depending on the molecular weight of the oligomer that has been targeted. Rather, the greater tan $\delta$ values exhibited by the very low Mwt oligomers would suggest that a system that contains these would likely to be bulk heated.

These observations were cross-referenced with the data in the authors prior publication, which compared in situ $\mathrm{MWH}$ and $\mathrm{CH}$ based synthesis of poly(methyl methacrylate) (PMMA) via CCTP [19]. In this paper, two different catalyst loadings (600 ppm and $270 \mathrm{ppm}$ ) were used to produce low molecular weight (Mwt) polymers. The results showed that when high catalyst load (600 ppm) were used, the final product were primarily dimer/trimer $\left(\mathrm{M}_{\mathrm{n}}=200\right.$ to $\left.300 \mathrm{~g} \mathrm{~mol}^{-1}\right)$ and the $\mathrm{MWH}$ and $\mathrm{CH}$ product polymer exhibited very similar Mwts. Meanwhile, when the catalyst level was reduced (270 ppm CTA added), a polymer of $\sim 700 \mathrm{~g} \mathrm{~mol}^{-1}$ (i.e. a chain length of approximately 7 monomer units) was isolated from the MWH reaction and Mwt differences in the product polymer were obtained between the MWH and $\mathrm{CH}$ methods.

By use of the new catalyst data reported in Table 1, these observations has been attributed to the fact that, in the experiments with the high catalyst loading where dimer/trimer were the product, the highest dielectric properties in the system are exhibited by the product dimmer/trimer and the initiated dimer/trimer (i.e. where the chain end of the polymer contains a radical) (shown in Table 1 Entry 3, 4 and 7). Therefore, it is proposed that this system is likely to be bulk heated as the energy is 
increasingly being put into the polymers rather than the catalyst as the degree of conversion to product increases, hence there is no difference between the $\mathrm{MWH}$ and $\mathrm{CH}$ experiments. However, once the catalyst level was dropped (270 ppm CTA added) it was observed that different Mwts were produced from the $\mathrm{MWH}$ and $\mathrm{CH}$ polymerisations $\left(\mathrm{M}_{\mathrm{n}}=670 \mathrm{~g} \mathrm{~mol}^{-1}\right.$ for the $\mathrm{MWH}$, and 200 to $300 \mathrm{~g} \mathrm{~mol}^{-1}$ for $\left.\mathrm{CH}\right)$. Table 1 shows that the dielectric properties of the oligomers drop as the Mwt increases, due to the greater restrictions on dipolar rotation imposed as the molecular structure becomes more complex. Thus, it was proposed that the molecular component that will exhibit the highest $\tan \delta$ values will be the initiated polymer in this system. This is because the radical, due to the chain growth nature of the free radical polymerisation mechanism, will be located at the chain end and so will undergo the least restriction to its movement and this exacerbated by the fact that it is a strong dipole. Thus, whilst the tan $\delta$ value of the initiated heptamer is predicted to drop from the value exhibited by the initiated dimer, this reduction is expected to be less than of the non-initiated equivalent. Unfortunately, a pure sample of the heptamer could not be isolated to ascertain the actual value due to its solid physical form. Thus, in the MWH reaction, the radical containing species will now be dielectrically dominant and so will be preferentially heated. As a result, the propagation (i.e. chain growth) process will be the one that is promoted (shown in Table 1 Entry 5, 6, 8 and 9) and the resulting products are mainly polymers with higher Mwts (i.e. heptamer). Meanwhile, the $270 \mathrm{ppm} \mathrm{CH}$ experiment is still bulk heating and so the same relative reactivates are in place and so the product Mwt remains unchanged. This conclusion was supported by the relative wields of the comparative $\mathrm{MWH}$ and $\mathrm{CH}$ reaction where the $\mathrm{MWH}$ promotion of the propagation reaction results in an additional $\sim 36 \%$ yield. This means that the designed catalyst does 
not possess dielectric characteristics of sufficient magnitude to ensure that chain transfer dominates the mechanism via being selectively heated.

\subsection{Microwave Heating Experiments}

A key aim of the present study was to define the affect that the presence of the catalyst/precursors have upon the heating and dielectric properties of the mixture at the outset of a MWH bulk polymerisation, i.e. one in which the monomer also acts as the solvent. In this region no polymerisation has occurred, thus the bulk will exhibit the viscosity of the monomer. Thus, cyclohexanone was chosen as a non-polymerising solvent for these test studies due to its; (a) ability to solubilise sufficient catalyst/precursors and enable the study to be conducted and (b) dielectric properties are similar to methyl methacrylate (MMA), which was the monomer/solvent that had been used in the prior reported bulk polymerisation studies. The physical properties of MMA and cyclohexanone are compared in Table 2, which shows that the $\tan \delta$ values are very similar compared to other common monomers/solvents, whilst confirming its greater polarity and hence solvating ability via its larger Debye value.

Table 2. Physical properties of solvents used in this study at $20^{\circ} \mathrm{C}$.

\begin{tabular}{ccc}
\hline Solvent / Monomer & $\begin{array}{c}\text { Loss tangent } \\
(\tan \delta)\end{array}$ & $\begin{array}{c}\text { Dipole moment } \\
\text { [Debye] }\end{array}$ \\
\hline methyl methacrylate & $0.08^{\mathrm{a}}$ & $1.79 \pm 0.26^{\mathrm{b}}$ \\
cyclohexanone & $0.12^{\mathrm{c}}$ & $2.87 \pm 0.04^{\mathrm{d}}$ \\
E-caprolactone & $0.7^{\mathrm{e}}$ & $3.6 \pm 0.2^{\mathrm{e}}$ \\
Ethanol & $0.941^{\mathrm{f}}$ & $1.69 \pm 0.05^{\mathrm{g}}$
\end{tabular}

a See reference [19], 'b See reference [22], ' See reference [23], 'd See reference[24], e See reference [25], ${ }^{f}$ See reference [26], see reference [27].

Following this assessment, a series of MWH experiments involving this solvent, both alone and when containing the precursors/catalysts (i.e. concentration of the catalyst /precursors in the solvents was $240 \mathrm{ppm}$ ), were conducted at a frequency of $2450 \mathrm{MHz}$ 
and $150 \mathrm{~W}$ constant input power. The aim was to define the factors that affected the MWH of catalyst/precursor containing solutions. The temperature and power profiles for these MWH trials are shown in Figure 4, where the reproducibility in the experimental measurement of input power was \pm 6 Watts and for all temperature measurements were less than $\pm 2{ }^{\circ} \mathrm{C}$.
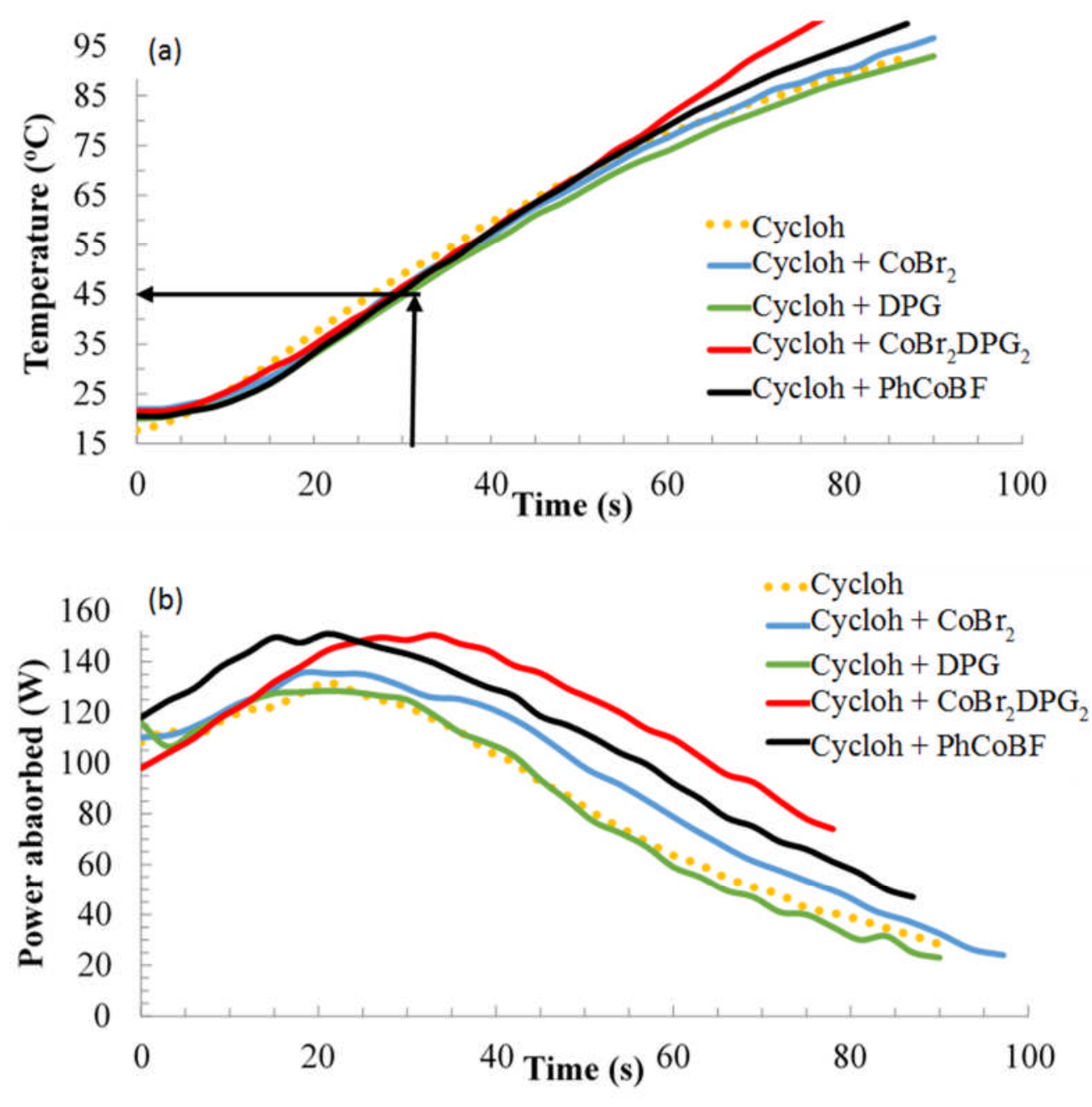

Figure 4. Profiles plots of the variation in (a) temperature and (b) power with time for cyclohexanone (cycloh) and cyclohexanone plus catalysts and/or precursors.

Figure 4(a) demonstrated that the heating profiles of the cyclohexanone and cyclohexanone/DPG solutions were very similar, indicating that a limited level of 
system heating resulted from the presence of the ligand precursor alone. By comparison, the solutions containing the halide precursor, premade PhCoBF or in situ synthesised organometallic catalysts were noted to produce measureable increases in the heating rate and achieved higher bulk temperatures than neat cyclohexanone. However, the profile of the organically liganded catalysts were significantly larger in both of these characteristics than that of the halide pre-cursor. Cross-referencing this data with the power profiles in Figure 4(b) showed that the ligand containing solution exhibited the same power absorption profile as the solvent. Meanwhile, those solutions containing organometallic species were found to absorb greater levels of power than the solvent alone. Table 3 contains a summary of more detailed data from Figure 4.

Table 3. Detailed data for heating experiments using cyclohexanone.

\begin{tabular}{cccccc}
\hline Entry & Catalyst & $\begin{array}{c}\text { Temperature } \\
\text { Overshoot } \\
\text { at } 240 \mathrm{ppm} \\
{\left[{ }^{\circ} \mathrm{C}\right]}\end{array}$ & $\begin{array}{c}\text { Additional } \\
\text { Absorbed } \\
\text { Power } \\
\text { at } 240 \mathrm{ppm} \\
{[\mathrm{W}]}\end{array}$ & $\begin{array}{c}\text { Temperature } \\
\text { Overshoot } \\
\text { at } 1100 \mathrm{ppm} \\
{\left[{ }^{\circ} \mathrm{C}\right]}\end{array}$ & $\begin{array}{c}\text { Additional } \\
\text { Absorbed } \\
\text { Power } \\
\text { at 1100ppm } \\
{[\mathrm{W}]}\end{array}$ \\
\hline $\mathbf{1}$ & $\mathrm{DPG}$ & $1 \pm 2$ & $1 \pm 6$ & $3 \pm 2$ & $8 \pm 6$ \\
$\mathbf{2}$ & $\mathrm{CoBr} 2$ & $4 \pm 2$ & $5 \pm 6$ & $13 \pm 3$ & $15 \pm 6$ \\
$\mathbf{3}$ & $\mathrm{PhCoBF}_{\mathbf{4}}$ & $11 \pm 2$ & $20 \pm 6$ & $16 \pm 5$ & $20 \pm 6$ \\
\hline
\end{tabular}

The halide precursor was noted to absorb approximately 5 Watts more than the neat solvent and those of both the premade and in situ catalysts absorb significantly more ( 20 Watts) than the solvent at the $240 \mathrm{ppm}$ level. Relating these data to the levels of energy input the cyclohexanone absorbs about $87 \%$ of the applied MW power at its maximum, but with the addition of the catalysts this rose to $99 \%$ of the MW power. Thus it is clear that the addition of the premade or in situ formed organic liganded catalysts significantly improves the MW absorption of the mixture. Again the more flexible bidentate $\mathrm{CoBr}_{2} \mathrm{DPG}_{2}$ was shown to outperform the PhCoBF due to its greater 
dipole mobility which resulted in enhancements in both the heating performance and final temperature being observed, for the same level of power absorbed.

Furthermore, whilst both premade $\mathrm{PhCoBF}$ and in situ $\mathrm{CoBr}_{2} \mathrm{DPG}_{2}$ were observed to absorb the same amount of energy, the time for maximum absorption for both catalysts was noted to be different. The premade catalyst (PhCoBF) was noted to achieve the maximum absorption approximately 18 seconds before the in situ equivalent. This delay was attributed to the need to synthesise the catalyst in the in situ case. This data would indicate that it takes only approximately 32 seconds to generate all the in situ catalyst. Additionally, this indicates that the additional $5 \mathrm{~W}$ absorbed by halide precursor sample, whilst insufficient to raise the bulk mixture temperature, is enough to promote the reaction between the precursor and the ligand to achieve the rapid creation of the catalyst. Furthermore, by cross referencing this to the temperature profile, it can be defined that the bulk temperature is only in the region of $45^{\circ} \mathrm{C}$ by the time that the catalyst has been synthesised (see black correlation arrows on Figure 4 (a)). This is well below the 1 hour half-life temperature of the initiator that has been used in the previously reported in situ polymerisation papers (2,2'-Azobis(2-methylpropionitrile) $($ AIBN $)-1$ hour half-life temp $=82^{\circ} \mathrm{C}$ ) [19]. This confirms that the catalyst will be synthesised and fully available to control the polymerisation prior to the polymerisation commencing.

Considering that catalyst concentrations were in parts per million (ppm) level, this level of additional system heating via the catalyst indicates that the intermolecular friction between organometallic complex and solvent would be significant in order to raise the temperature of the bulk solvent by this much. To confirm that this heating rate differential was due to the present of the organometallic species, a second series of heating trials were conducted where the organometallic concentration was raised to 
$1100 \mathrm{ppm}$. These experiments exhibited an increased rate of heating, achieved a higher final temperature and the differential between the level of the trend in the $\mathrm{CoBr}_{2} \mathrm{DPG}_{2}$ was increased (see Table 3 and Figure S1 in the ESI document). This data confirmed that the differences in the heating data were linked to the organometallic species present and that the designed, more flexible in situ catalyst showed greater differentiation from the more rigid $\mathrm{PhCoBF}$ as concentration increased in $\mathrm{MWH}$.

To investigate the effect of ligand structure further, a second in situ catalyst was prepared containing the dimethylglyoxime ligand (DMG). In this case the ligand's phenyl functional groups are replaced by methyl groups. The MWH data comparison with the DPG complex is shown in Figure 5.
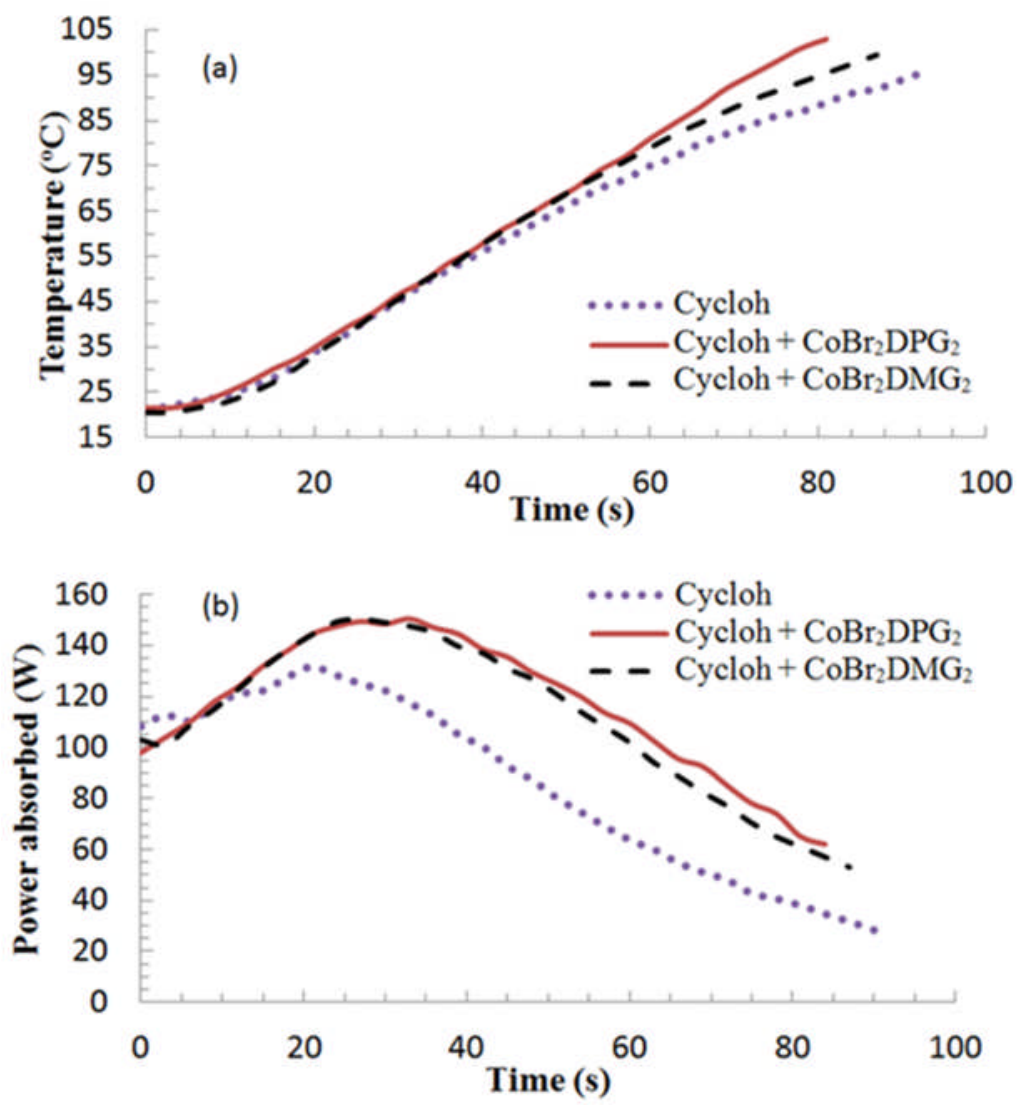

Figure 5. Profiles plots of the variation in (a) temperature and (b) power with time for cyclohexanone (cycloh) and cyclohexanone plus $\mathrm{CoBr}_{2} \mathrm{DPG}_{2}$ and $\mathrm{CoBr}_{2} \mathrm{DMG}_{2}$. 
The DMG ligand was expected to be more flexible than the phenyl version because of the reduced steric hindrance associated with the bulky aromatic groups. However, Figure 5 showed that the DMG complex was observed to exhibit lower heating characteristics than the DPG equivalent empirically. Thus, it was proposed that the phenyl functionality must produce greater solvation and/or a greater level of dipole/EM field interactions within the DPC ligand, which leads to the phenyl complexes outperforming the methyl version. Thus the design of MWH responsive complexes will clearly require a balancing of a number of key molecular factors.

Across all samples, it was noticed that the power absorption depleted as the experiment proceed. This is because the temperature of the solution is increasing as the heating is on-going (see Figure 4 (a)). As the temperature increases so the tan $\delta$ has been observed to decrease (see Figure 2) and hence the level power absorbed per unit time is reduce as the energy already with the sample is not bring dissipated so rapidly. This reduction was also attributed to the reduction in viscosity/density of the medium as temperature increase, which in turn reduces the level of intermolecular interaction that each molecule experiences.

\section{Conclusion}

This study demonstrates that it is possible to molecularly design a catalyst to be more susceptible to selective MWH and that; (a) to accomplish this, there are a number molecular characteristics that potentially have to be balanced to achieve this increased dielectric performance and (b) EM dielectric properties of the catalyst have to be amongst, if not the most, dominant values of all the species within the mixture if it is to drive the mechanistic chemistry within a reaction. 
The investigation into the effect of selective MWH on the synthesis and use of two designed in situ catalysts has shown that: (a) adding the organometallic compounds/catalysts to a solvent at the ppm level produces measureable increases in the rate of heating exhibited by a solution containing them, (b) clearly demonstrated that changing the ligand system could influence the solutions ability to contribute to the heating of the bulk mixture/promote a chemical transformation and (c) supported the conclusion that the in situ catalyst is synthesised in approximately 30 seconds. The data also showed that, whilst there is enough EM power absorbed by the $\mathrm{CoBr}_{2} /$ cyclohexanone solution to drive the in situ complexation reaction, this level of energy is not enough to contribute significantly to the heating of the overall mixture. Thus, these observations suggest that it is possible to drive chemical reactions with low levels of EM energy without significantly heating the bulk mixture.

In this case, the difference in heating behaviour between the neat solvent/solvent plus ligand mixture and those solutions containing the metal complexes was attributed to the greater dielectric loss factor values exhibited by the organometallic containing solutions. Of these solutions, these possessing the organically liganded catalyst were observed to heat most significantly and it was proposed that the greater solubility and dipole content/type (e.g. adding organic ligands, converting a single tetradentate ligand to two bidentate species, or introducing different functional groups onto the ligand) influences their ability to translate absorbed energy into molecular motion, by affecting its relaxation process and so produce heat/drive chemical transformations. Furthermore, cross referencing the timescale for this catalyst synthesis with the temperature profiles of a typical CCTP polymerisation would also suggest that the catalyst is synthesised prior to the polymerisation initiating and so it is able to fully control the process. Thus, 
it does provide a significant opportunity to intensify current industrial process with the application of MWH.

The data also shows that the level of selective heating in a polymerisation to produce oligomers is dependent on the molecular weight of the product oligomer. Furthermore, the response of the catalyst designed in this study is likely to be less than that of the growing polymer chain. Thus for the manufacture of oligomers with this currently designed catalyst is not sufficiently EM active to dominate the process, Rather it is the propagation step that is the reaction that is promoted by selective heating, so explaining and so explains why longer chain length result from the MWH reactions.

\section{Acknowledgements}

The authors wish to thank Dr Kevin Adlington for his help with the experimental activities and the Microwave Process Engineering (MPE) group at the University of Nottingham for the financial support of the present work.

\section{References}

1. Julien Nicolas, Yohann Guillaneuf, Catherine Lefay, Denis Bertin, Didier Gigmes, and Bernadette Charleux. Progress in Polymer Science 2013, 38, p. 63-235, DOI: 10.1016/j.progpolymsci.2012.06.002.

2. Krzysztof Matyjaszewski, Jianhui Xia. Chemical Review 2001, 101, p. 2921-2990, DOI: $10.1021 /$ cr940534g.

3. Sebastien Perrier, Pittaya Takolpuckdee. Journal of Polymer Science Part A:

Polymer Chemistry 2005, 43, p. 5347-5393, DOI: 10.1002/pola.20986.

4. Alexei Gridnev, Steven Ittel. Chem. Rev. 2001, 101, p. 3611-3659, DOI: $10.1021 /$ cr9901236. 
5. Papia Haque, Ian A. Barker, Andrew Parsons, Kristofer J. Thurecht, Ifty Ahmed, Gavin S. Walker, Christopher D. Rudd, Derek J. Irvine. J. Polym. Sci., Part A: Polym. Chem. 2010, 48, p. 3082-3094, DOI: 10.1002/pola.24086.

6. Gordon D. Airey, Jasmin Wilmot, James R.A. Grenfell, Derek J. Irvine, Ian A. Barker, Jaouad El Harfi. Eur. Polym. J. 2011, 47, p. 1300-1314, DOI: 10.1016/j.eurpolymj.2011.03.002.

7. Jianing Li, Jaouad El harfi, Steven M. Howdle, Kim Carmichael, Derek J. Irvine. Polymer Chemistry 2012, 3, p. 1495-1501, DOI: 10.1039/C2PY20066J.

8. Alexei Gridnev. Journal of Polymer Science Part A: Polymer Chemistry 2000, 38, p. 1753-1766, DOI: 10.1002/(SICI)1099-0518(20000515)38:10<1753::AIDPOLA600>3.0.CO;2-O.

9. K. G. Suddaby, D. M. Haddleton, J. J. Hastings, S. N. Richards, J. P. O'Donnell. Macromolecules 1996, 29, p. 8083-8091, DOI: 10.1021/ma960528h.

10. Kevin Adlington, Robert McSweeney, Georgios Dimitrakis, Samuel W.

Kingman, John P. Robinson, Derek J. Irvine. RSC Adv. 2014, 4, p. 16172-16180, DOI: 10.1039/C4RA00907J.

11. Kevin Adlington, Anthony Green, Wenxin Wang, Steven M. Howdle, Derek J. Irvine. Dalton Trans. 2013, 42, p. 127-136, DOI: 10.1039/C2DT31822A.

12. David E Clark, Diane C Folz, Jon K West. Mat. Sci. Eng. A 2000, 287, p. $153-$ 180, DOI: 10.1016/S0921-5093(00)00768-1.

13. D. A. Jones, T. P. Lelyveld, S. D. Mavrofidis, S. W. Kingman, N. J. Miles. Resources, Conservation and Recycling 2002, 34, p. 75-90, DOI: 10.1016/S09213449(01)00088-X.

14. Erdan Hu, Hefa Cheng. American Chemical Society 2013, p. 533-541, DOI: 10.1021/es303486j. 
15. Erdan Hu, Hefa Cheng. Water Research 2014, 57, p. 8-19, DOI: 10.1016/j.watres.2014.03.015.

16. Nam T. Nguyen, Edward Greenhalgh, , Kim Carmichael, Georgios Dimitrakis, Samuel W. Kingman, John P. Robinson, Derek J. Irvine. Tetrahedron 2014, 70, p. 996-1003, DOI: 10.1016/j.tet.2013.11.031.

17. Alstair D. Smith, Edward Lester, Kristofer J. Thurecht, Jaouad El Harfi, Georgios Dimitrakis, Sam W. Kingman, John P. Robinson, and Derek J. Irvine. Industrial \& Enginerering Chemistry Research 2010, 49, p. 1703-1710, DOI: 10.1021/ie901201h.

18. Alstair D. Smith, Edward Lester, Kristofer J. Thurecht, Sam W. Kingman, Jaouad El Harfi, Georgios Dimitrakis, Jaouad El Harfi, Derek J. Irvine. Industrial \& Enginerering Chemistry Research 2010, 49, p. 3011-3014, DOI: 10.1021/ie901389a.

19. Kevin Adlington, G. Joe Jones, Jaouad El Harfi, Georgios Dimitrakis, Alastair Smith, Sam W. Kingman, John P. Robinson, Derek J. Irvine. Macromolecules 2013, 46, p. 3922-3930, DOI: $10.1021 / \mathrm{ma} 400022 y$.

20. Camelia Gabriel, Sami Gabriel, Edward H. Grant, Ben S. J. Halstead, D. Michael P. Mingos. Chem. Soc. Rev. 1998, 27, p. 213-223.

21. Sebastian Losse, Johannes G. Vos, Sven Rau. Coordination Chemistry Reviews 2010, 254, p. 2492-2504, DOI: 10.1016/j.ccr.2010.06.004.

22. J. E. Salz, P. Hummel, P. J. Flory. Journal of Physical Chemistry 1981, 85, p. 3211, DOI: $10.1021 / \mathrm{j} 150622 \mathrm{a} 002$.

23. R. J. Meakin. Transactions of the Faraday Society 1962, 58, p. 1905 - 1971, DOI: $10.1039 /$ TF9625801962.

24. Yoshitake Ohnishi, Kunio Kozima. Bulletin of the Chemical Society of Japan 1968, 41, p. 1323-1325, DOI: http://doi.org/10.1246/bcsj.41.1323. 
25. Ronald Hutcheon, Jozef Mouris, Xiaomei Fang, Daniel A Scola, Microwave Properties North (MPN). CERAMIC TRANSACTIONS 2001, 111, p. 109-118.

26. C. Oliver Kappe, Alexander Stadler. 2006, Wiley-VCH Verlag GmbH \& Co. KGaA. p. 9-28, DOI: 10.1002/3527606556.ch2.

27. Seong-Jeen Kim, Sang-Hoon Lee, Cheol-Jin Lee. Journal of physics d: Applied physics 2001, 34, p. 3505-3509, DOI: http://dx.doi.org/10.1088/0022$3727 / 34 / 24 / 314$ 